\title{
Nano-bio-optomechanics: nanoaperture tweezers probe single nanoparticles, proteins, and their interactions
}

\section{Reuven Gordon}

Reuven Gordon, "Nano-bio-optomechanics: nanoaperture tweezers probe single nanoparticles, proteins, and their interactions," Proc. SPIE 9544, Metamaterials, Metadevices, and Metasystems 2015, 954403 (1 September 2015); doi: 10.1117/12.2193785

Event: SPIE Nanoscience + Engineering, 2015, San Diego, California, United States 


\title{
Nano-Bio-Optomechanics: Nanoaperture Tweezers Probe Single Nanoparticles, Proteins, and their Interactions
}

\author{
Reuven Gordon \\ Department of Electrical and Computer Engineering, University of Victoria, \\ Victoria, BC, Canada, V8P5C2
}

\begin{abstract}
Nanoparticles in the single digit nanometer range can be easily isolated and studied with low optical powers using nanoaperture tweezers. We have studied individual proteins and their interactions with small molecules, DNA and antibodies. Recently, using the fluctuations of the trapped object, we have pioneered a new way to "listen" to the vibrations of nanoparticles in the $100 \mathrm{GHz}-1 \mathrm{THz}$ range; the approach is called extraordinary acoustic Raman (EAR). EAR gives unprecedented low frequency spectra of individual proteins in solution, allowing for identification and analysis, as well as probing their role in biological functions. We have also used EAR to study the elastic properties, shape and size of various individual nanoparticles.
\end{abstract}

Keywords: Optical Tweezers, Optomechanics, Nanoparticles, Biosensors, Plasmonics

\section{INTRODUCTION}

Nanoparticles in the size range of 1-50 $\mathrm{nm}$ are important building blocks for technology and biology. DNA, proteins, protein complexes, antibodies and viruses are biological nanoparticles in this size range. Quantum dots, nanocarbon and colloidal nanoparticles are among the technologically relevant nanoparticles similarly sized. Our aim is to develop new tools to understand such nanoparticles at the single particle level.

We are looking at approaches to hear the acoustic vibrations of nanoparticles. What does a protein sound like and how does this relate to its functioning? What happens when the protein is a mutant form? Does it sound "sick"? If so, can we use this as a diagnostic or as an assay to discover drugs?

Acoustic modes of proteins have been studied in detail using various theoretical approaches; of particular note, molecular dynamics simulations [1]. While these theoretical approaches are valuable to better understand protein function and their interactions (for example, for applications in drug discovery), there are limited experimental approaches to validate such models. Raman (and its relative, Brillouin scattering) has been used to probe the acoustic vibrations of nanoparticles. For example, C60 (Buckminsterfullerene) has Raman active vibrations at $273 \mathrm{~cm}^{-1}$ and 497 $\mathrm{cm}^{-1}$. For larger nanoparticles, the typical vibration frequencies start below $100 \mathrm{GHz}$ (around $3 \mathrm{~cm}^{-1}$ ). This is a challenge for Raman due to the Rayleigh line overwhelming the weak Raman signal, and also for micro-Brillouin due to limited scattering for nanoparticles below $200 \mathrm{~nm}$. Ideally, we would like a method to probe individual nanoparticle vibrations in the range from $10 \mathrm{GHz}$ to $1 \mathrm{THz}$. For proteins in particular, the vibrational density of states is relatively sparse in this range, and so it is promising for analysis. This can be compared with typical vibration frequencies founding in conventional optomechanics of micron-scale structures; which rarely reaches to $\mathrm{GHz}$ frequencies due to the larger size (although there are fairly recent examples at $5 \mathrm{GHz}[2]$ ).

Single nanoparticle/molecule studies have been developed for just over a decade. Of note, single molecule fluorescence approaches developed by W. E. Moerner and co-workers were recognized with the 2014 Nobel Prize in Chemistry [3]. There are many reasons to attempt single nanoparticle studies, including:

- removing heterogeneity from a population,

- seeing richer statistics (non-averaged behavior),

- studying dynamic processes without the need for synchronization,

- studying nanoparticles over a wide range of concentrations, and

- sensing vary rare particles/events.

Metamaterials, Metadevices, and Metasystems 2015, edited by Nader Engheta,

Mikhail A. Noginov, Nikolay I. Zheludev, Proc. of SPIE Vol. 9544, 954403

(C) 2015 SPIE $\cdot$ CCC code: $0277-786 X / 15 / \$ 18 \cdot$ doi: $10.1117 / 12.2193785$

Proc. of SPIE Vol. 9544 954403-1 
Even with highly innovative and impactful developments using single molecule fluorescence approaches, there are still drawbacks to existing methods. For example, a tour de force 2012 study of protein folding using Förster resonance energy transfer at the single protein level illustrates the challenges [4]:

1. Adding fluorescent markers alters the native protein, so there is always the question about whether the marker is influencing the actual biophysical process.

2. A tether is usually used to immobilize the protein for study (otherwise it would diffuse away).

3. Fluorescence can be a relatively slow process, so photon counting is required to resolve faster dynamics and usually the molecule has to be pumped quite "hard" to ensure enough signal is achieved. Even so, glycerol needs to be used to slow down the protein folding [4].

4. The fluorophores bleach, so observation time is limited.

5. The signal observed is open to interpretation since it is coming from an exogeneous marker, not the protein itself.

Despite all these challenges, single molecule fluorescence approaches have advanced significantly our understanding of biological processes. Even so, it is highly desirable to develop methods that are not subject to these limitations. In particular, we would like a single nanoparticle detection platform that has the following features:

- $\quad$ No labels/markers/fluorophores

- No tethers

- Fast response

- Extended observation times

- Direct measurement of nanoparticle (not an exogeneous signal open to interpretation)

- Fairly agnostic to concentration (can isolate single particles in dilute or dense mixtures)

Since 2009, we have been developing laser tweezers using apertures in metal films to study nanoparticles down to $50 \mathrm{~nm}$ [5]. In 2011, we had a breakthrough in this approach to allow trapping of much smaller particles by using the double nanohole (DNH) aperture shape, with two sharp cusps where the holes overlap, allowing for trapping of $12 \mathrm{~nm}$ dielectric nanoparticles [6], and then single proteins [7]. Even in the first single protein studies, we observed protein conformal changes [7], which showed the potential of this work to observe directly dynamics in individual proteins without labels or tethers at timescales that are limited by the detector response (typically into the GHz). Recently, however, we have developed this approach further to modulate resonantly the nanoparticles at their vibration frequency and thereby probe their dynamics at $\sim 100 \mathrm{GHz}$ [8]. As mentioned previously, this is exactly the regime where nanoparticles in the 1 to 50 $\mathrm{nm}$ size range start to show vibrational resonances, and so it is of interest for identifying them and understanding their behavior.

\section{DOUBLE NANOHOLE OPTICAL TWEEZERS}

Even the first works on gradient-force optical tweezers recognized the size-dependent limitation of Rayleigh scattering, requiring extremely large intensities to hold onto non-resonant particles below $100 \mathrm{~nm}$ [9]. The third power scaling of the polarizability for Rayleigh scatterers means that 1000 times more intensity is required to have the same force on a $10 \mathrm{~nm}$ particle as on a $100 \mathrm{~nm}$ particle.

Our trick to overcome this strong size-scaling limitation was to use an aperture in a metal film. Like nanoparticles, nanoapertures also have strong size scaling dependence: their transmission depends on the ratio between the aperture size and the wavelength to the fourth power according to Bethe's theory. Adding a dielectric material into the aperture effectively reduces the wavelength or makes the aperture appear larger. Therefore, there is a strong change in the transmission due to the strong scaling from Bethe's theory, even though the nanoparticle is a Rayleigh scatterer. We used this nanoaperture to observe optical trapping; when a particle enters the aperture, there is a jump in the optical 
transmission [5]. The particle is kept in the aperture by the resulting optical forces, as was verified by a maintained higher level of transmission. The transmission dropped if the particle escaped. Also, multiple particle trapping events could be distinguished from single particle trapping events (all showing the same step height), but a distinct second step in the transmission, as shown in Figure 1. The trapping of multiple particles is rare for typical experimental configurations used, which we believe is due to steric hindrance, repulsion of like-charged particles, and the improbability of such coincidences (at lower concentrations).

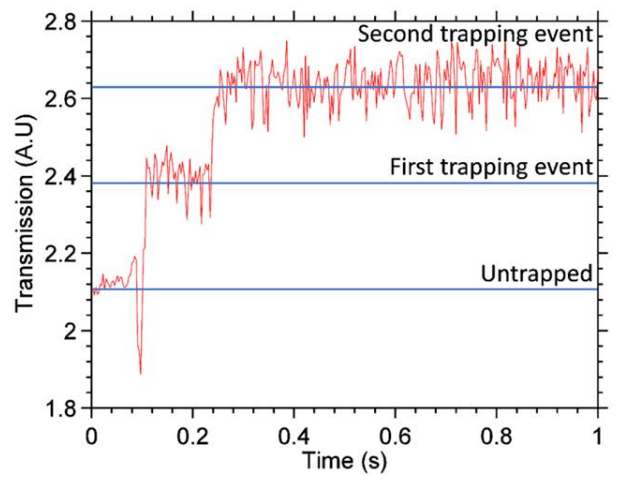

Figure 1. Trapping of two identical nanoparticles in sequence showing two steps in the transmission of almost the same size.

(Single nanoparticle trapping events show a single step equal to the height of the first trapping event).

It should be noted that others had considered the use of apertures in optical tweezer systems prior to our work, both theoretically [10] and in experiment [11], yet we are unaware of any previous works that exploited this effect to hold onto nanoparticles smaller than $\sim 100 \mathrm{~nm}$.

While a cylindrical aperture was able to trap nanoparticles down to $\sim 50 \mathrm{~nm}$, going to smaller sizes required shaped apertures. In particular, we used the double nanohole (DNH), which we studied since 2004 [12-14] because it creates a local hot-spot of around $10 \mathrm{~nm}$ in size in the electric field that interacts more strongly with the nanoparticle of interest. With the DNH, we were able to trap $12 \mathrm{~nm}$ silica spheres [6], single proteins [7], and 20 bases of single stranded DNA [15], among other things. More recently, researchers have started to use bowtie nanoapertures [16]; these share the same features of the DNH structure that enable trapping down to the size of single proteins - in particular, sharp cusps with a narrow gap where two holes overlap.

\section{PROTEINS, DNA AND THEIR INTERACTIONS}

The ability to trap single proteins has allowed for studying protein-antibody binding [17], protein-DNA interactions [15], protein sizing [18], protein-protein interactions [18], protein small-molecule interactions (both in the strong binding [19] and weak binding limits [20]). In typical studies, the aperture transmission is used to monitor light scattering changes by the protein or DNA particles, thereby giving direct information about their structure (via their diffractive properties).

The ability to directly monitor proteins and their interactions is of obvious interest for drug discovery applications. Not only is it possible to see directly if a small molecule is binding to a protein (through changes in the fluctuations of the optical transmission through the aperture - representing thermal motion of the particle) [19], but it is also possible to monitor on-off binding kinetics to determine the binding affinity [20]. In this way, potential small molecule drug candidates can be probed for their interactions with proteins. Because this approach operates at the single molecule level, it has the potential to conserve valuable protein sample.

One of the interesting systems we studied with this approach is the so-called tumor suppressor protein, p53 [15]. This protein is reported to be mutated in the majority of known cancers. It is well known that p53 binds to DNA; however, the mutant form can also bind with almost the same affinity. To probe the function of p53, we first trapped a hair-pin of single-stranded DNA (ssDNA) that binds with p53. This hairpin is unzipped by electrostriction in the DNH trap: the unzipped ssDNA is longer and has a higher polarizability; therefore, its dipole interaction with the local electric field is greater. In the presence of p53, the unzipping time of the hairpin is increased by orders of magnitude. Interestingly, a point mutation to $\mathrm{p} 53$, which still binds strongly to the hairpin, does not increase the unzipping time. Therefore, our 
experiment has shown that p53 acts to suppress the unzipping of DNA and that it cannot perform this task with a common mutation. (We also quantified the energy barrier to unzipping that is created by wild-type p53 binding using Arrhenius scaling). This approach may be used as an assay to screen for drugs that could correct the function of p53, and possibly play an important role in suppressing the development cancers.

\section{EXTRAORDINARY ACOUSTIC RAMAN}

As described above, the DNH optical tweezer pulls on ssDNA in the trap through electrostriction. If we modulate the intensity of the trapping laser beam, then the electrostriction is also modulated and it is possible to drive vibrations in the ssDNA. When this is done, we observed increased amplitude fluctuations of the laser transmission for certain driving frequencies. This increased root mean squared (RMS) amplitude fluctuation is attributed to hitting a resonance of the trapped ssDNA, heating it up, and thereby producing an increase in thermal motion. By scanning the frequency, a spectrum of the vibrational resonances of the ssDNA can be achieved (see Figure 2). We noted changes in the resonance frequency with length of the ssDNA and found good agreement with a linear chain oscillator model [21].
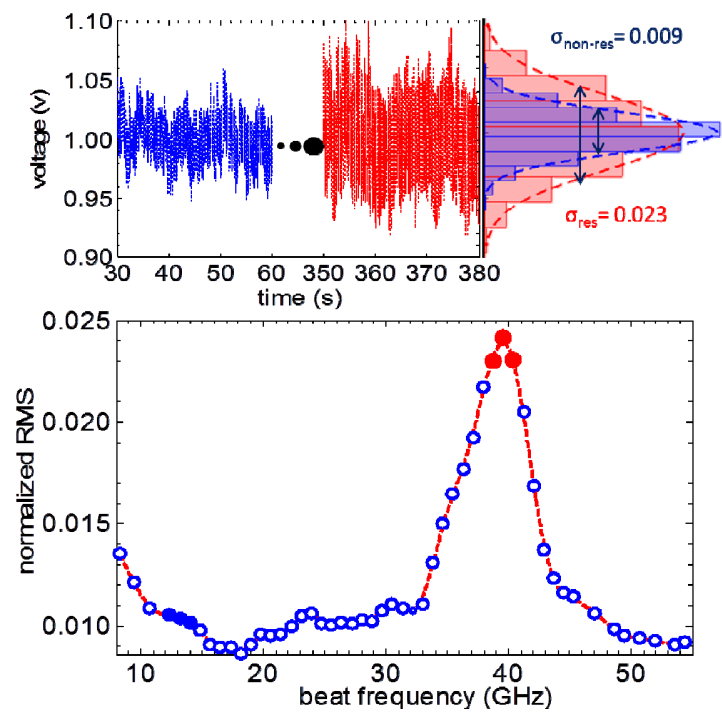

Figure 2. Increased noise amplitude (RMS fluctuations) observed when intensity modulation hits resonance frequency of ssDNA. Top shows increase in noise and bottom shows noise amplitude over a range of modulation frequencies.

We referred to this process as extraordinary acoustic Raman (EAR) because of its single molecule sensitivity and ability to access low wavenumbers with high resolution. Unlike related coherent types of spectroscopy, like the optical Kerr effect, here we measure the random motion of the particle, so we are not as sensitive to energy redistribution that is common in proteins [1]. Figure 3 shows the spectrum taken for a common protein. Other examples can be found elsewhere [8]. Using this approach, we have also measured the vibration spectra of the MS2 bacteriophage (virus).

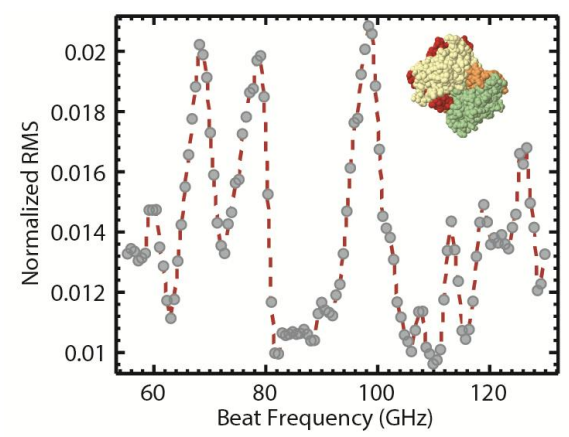

Figure 3. EAR spectrum of a single protein in the DNH trap. 


\section{TECHNOLOGICAL DEVELOPMENT OF DNH TWEEZER SYSTEMS}

Every nanotechnology faces the challenge of being able to achieve low-cost, high-throughput and reliable fabrication. Furthermore, it would be ideal to integrate the DNH optical tweezer system into a fiber-optic platform to improve scalability. First steps have been taken to solve the nanofabrication challenge by using a process of template stripping [22], where gap sizes down to $7 \mathrm{~nm}$ were produced. We have also integrated DNH structures on the ends of cleaved optical fibers and demonstrated trapping with those structures [23]. This is promising for future scalability where it is possible envision having multiple trapping fibers to each address a single well of a microwell plate, and thereby integrate seamlessly with existing biochemistry laboratory protocols. It should be noted that past works have also integrated bowtie nanoapertures on the end of tapered fibers and shown the ability to translate the trapped object [16]; however, care should be taken to avoid damaging the tapered fiber, and the tapering process complicates the fabrication.

\section{CONCLUSIONS AND OUTLOOK}

The DNH optical tweezer approach allows us to study single proteins and other nanoparticles. Unlike fluorescence based methods, the DNH approach is label-free (uses the intrinsic light scattering properties of the nanoparticle) and does not require tethering to prevent the particle from diffusing away. We have already studied protein sizes, protein-protein interactions, protein-small molecule interactions, protein antibody interactions, protein-DNA interactions with this approach. Adding a second trapping laser of different frequency allows for exciting vibrations in the nanoparticle of interest, which can be used to build the acoustic Raman spectrum of the nanoparticle by sweeping the laser frequency difference in the range of $\sim 100 \mathrm{GHz}$ (extremely high frequency to $\mathrm{THz}$ bands). In this way, it is possible to identify individual proteins, as well as study the properties of nanoparticles (such as material anisotropy).

We are developing this method in terms of scalability and ease of nanofabrication to allow for widescale adoption in the areas of drug discovery, diagnosis and basic scientific analysis.

\section{ACKNOWLEDGEMENTS}

RG thanks Skyler Wheaton for the data in Figure 1 and 3 and Abhay Kotnala for the data in Figure 2. Funding from the Natural Sciences and Engineering Research Council (Canada) Discovery Grants program is acknowledged.

\section{REFERENCES}

[1] Nicolaï, A., Delarue, P., Senet, P. "Low-Frequency, Functional, Modes of Proteins: All-Atom and Coarse-Grained Normal Mode Analysis" in Computational Methods to Study the Structure and Dynamics of Biomolecules and Biomolecular Processes. Springer Berlin Heidelberg, 483-524, (2014).

[2] Chan, J., Safavi-Naeini, A. H., Hill, J. T., Meenehan, S., Painter, O. "Optimized optomechanical crystal cavity with acoustic radiation shield," Applied Physics Letters 101(8), 081115 (2012).

[3] Moerner, W. E. "Single-Molecule Spectroscopy, Imaging, and Photocontrol: Foundations for Super-Resolution Microscopy (Nobel Lecture)," Angewandte Chemie Advanced Online (2015).

[4] Chung, H. S., McHale, K., Louis, J. M., Eaton, W. A. "Single-molecule fluorescence experiments determine protein folding transition path times," Science 335(6071), 981-984 (2012).

[5] Juan, M. L., Gordon, R., Pang, Y., Eftekhari, F., Quidant, R. "Self-induced back-action optical trapping of dielectric nanoparticles," Nature Physics 5, 915-919 (2009).

[6] Pang, Y., Gordon, R., "Optical Trapping of $12 \mathrm{~nm}$ Dielectric Spheres Using Double-Nanoholes in a Gold Film," Nano Letters, 11 (9), 3763-3767 (2011).

[7] Pang, Y., Gordon, R. “Optical trapping of a single protein,” Nano Letters 12(1), 402-406 (2012).

[8] Wheaton, S., Gelfand, R. M., Gordon, R. "Probing the Raman-active acoustic vibrations of nanoparticles with extraordinary spectral resolution," Nature Photonics 9, 68-72 (2015). 
[9] Ashkin, A., Dziedzic, J. M., Bjorkholm, J. E., Chu, S. "Observation of a single-beam gradient force optical trap for dielectric particles,” Optics Letters, 11(5), 288-290 (1986).

[10] Okamoto, K., Kawata, S. "Radiation force exerted on subwavelength particles near a nanoaperture," Physical Review Letters 83(22), 4534 (1999).

[11] Kwak, E.-S., Onuta, T.-D., Amarie, D., Potyrailo, R., Stein, B., Jacobson, S. C., Schaich, W. L., Dragnea, B. "Optical trapping with integrated near-field apertures," The Journal of Physical Chemistry B 108(36) 13607-13612 (2004).

[12] Gordon, R., Hughes, M., Leathem, B., Kavanagh, K. L., Brolo, A. G. "Basis and Lattice Polarization Mechanisms for Light Transmission through Nanohole Arrays in a Metal Film," Nano Letters, 5, 1243-1246 (2005).

[13] Lesuffleur, A., Kumar, L. K. S., Brolo, A. G., Kavanagh, K. L., Gordon, R. "Apex-Enhanced Raman Spectroscopy Using Double-Hole Arrays in a Gold Film,” J. Phys. Chem. C, 111, 2347-2350 (2007).

[14] Min, Q., Santos, M. J. L., Girotto, E. M., Brolo, A. G., Gordon, R. "Localized Raman Enhancement from a DoubleHole Nanostructure in a Metal Film,” J. Phys. Chem. C, 112(39), 15098-15101 (2008).

[15] Kotnala, A., Gordon, R. "Double nanohole optical tweezers visualize protein p53 suppressing unzipping of single DNA-hairpins," Biomedical Optics Express 5(6), 1886-1894 (2014).

[16] Quidant, Nature Nano.

[17]Zehtabi-Oskuie, A., Jiang, H., Cyr, B., Rennehan, D. Al-Balushi, A., Gordon, R. "Double nanohole optical trapping: Dynamics and protein-antibody co-trapping," Lab Chip 13, 2563-2568 (2013).

[18] Wheaton, S., Gordon, R. "Molecular Weight Characterization of Globular Proteins using Optical Nanotweezers," Analyst 140, 4799 - 4803 (2015).

[19] Al Balushi, A. A., Gordon, R. "Label-Free Free-Solution Single-Molecule Protein.Small Molecule Interaction Observed by Double-Nanohole Plasmonic Trapping," ACS Photonics, 1(5), 389-393 (2014).

[20] Al Balushi, A. A., Gordon, R. "A Label-Free Untethered Approach to Single-Molecule Protein Binding Kinetics," Nano Letters 14 (10), 5787-5791 (2014).

[21] Kotnala, A., Wheaton, S., Gordon, R. "Playing the notes of DNA with light: extremely high frequency nanomechanical oscillations," Nanoscale 7, 2295-2300 (2015).

[22]Zehtabi-Oskuie, A., Zinck, A., Gelfand, R. Gordon, R. "Template stripped double nanohole in a gold film for nanooptical tweezers," Nanotechnology 25, 495301 (2014)

[23] Gelfand, R. M., Wheaton, S., Gordon, R. "Cleaved Fiber Optic Double Nanohole Optical Tweezers for Trapping Nanoparticles," Optics Letters 39(22), 6415-6417 (2014). 